\title{
An energy harvester with Two degrees of freedom Nonlinear oscillations
}

\author{
Zuyao Wang \\ School of Sciences, Zhejiang University of Science and Technology, Hangzhou Zhejiang 310023, \\ P.R. China \\ wangzuyao2001@163.com
}

\begin{abstract}
Keywords:Nonlinear vibration; magnetic levitation; energy harvesting; harmonic balance, numerical simulation

Abstract. This paper investigates a design of a energy harvester that uses magnetic levitation to produce an oscillator with a appendage linear spring. The harmonic balance method is developed to analyze the frequency-amplitude response relationship. The numerical results show that the strength and bandwidth of energy harvesting are improved by adjusting the mass ratio. The analytical results are supported by the numerical solutions.
\end{abstract}

\section{Introduction}

Harvesting surrounding waste kinetic energy to power low-powered electronics has emerged as a prominent research area and continues to grow rapidly[1]. Vibration-based energy harvesters are able to scavenge wasted vibrations from environment and converting wasted vibrations into electrical energy to power small-scale electronic device. Such technology provides a promising future for the development of self-powering sensors.As a promising approach, the piezoelectricity has been used to convert ambient vibration into useful electrical energy[2]. There are several excellent and comprehensive survey papers, notably Sodano [3], Anton and Sodano[4], and Tang et al. [5], reviewing the state of the art in different time phases of investigations related to piezoelectric energy harvesting. Recently, nonlinearity and bistability have been introduced to increase the operating frequency range of energy harvesters. Although the cantilevered piezoelectric energy harvester has been investigated most frequently, its output power and working bandwidth are still too small for practical use. Recently, magneto electric laminate composites (MLCs) [6] have been used to create vibration-based energy harvesters. Due to the high energy density and strong magneto-mechanical coup ling in magnetostrictive material, MLC has the potential to generate large power outputs. Dai et al. [7] designed an energy harvester using Terfenol-D/PZT/Terfenol-D laminate, which can produce a power at $71 \mathrm{~Hz}$ for a base acceleration. Their energy harvester was able to drive wireless sensor nodes at a certain communication distance and operation cycle. Wen-An Jiang et al [8-12] proposed energy harvesting of monostable Duffing oscillator under Gaussian white noise excitation.Zhu et al. [13-14] used Terfenol-D/PZT/Ter-fe nol-D laminate to harvest energy from nonlinear vibration created from magnetic levitation.

On the base of the design and analysis of a new magneto electric energy harvester, This paper investigates a design of a energy harvester that uses magnetic levitation to produce an oscillator with a appendage linear spring. The harmonic balance method is developed to analyze the frequency-amplitude response relationship.

\section{Governing Equation of the System}

A schematic diagram of the complete energy harvesting device is shown in Fig. 1. The device consists of the magnetic levitation system and a coil fabricated from seven layers of 36 guage enamelcoated copper wire that was wound around the outer casing. To describe the model for electromagnetic induction, we refer to the schematic diagram of Fig. 1 where two reference frames have been applied. The first reference frame is fixed in space and is used to describe the motion 
amplitude, $\mathrm{A}$, and excitation frequency, $\Omega$, of the outer housing $\mathrm{z}=\mathrm{A} \cos \Omega \mathrm{t}$. The second reference frame, designated as $\mathrm{x}$, describes the motion of the center magnet.The coil magnetic force is assumed to be proportional to the relative velocity between the center magnet and outer housing.It can be seen that both the magnetic force and field density depend on the relative displacement of the central magnet, which can be solve from the system's governing equation. By taking into account the nonlinear magnetic force, the force of gravity, appendage linear spring and the effect of damping, the governing equation of the system can be derived based on the Newton's second law as

$$
\left\{\begin{array}{c}
m_{1}\left(c_{1} x+k_{3} x^{3}+m_{1} a-c_{2}-k_{2} y=0\right. \\
m_{2}\left(c_{2}+k_{2} y+m_{2} a=0\right.
\end{array}\right.
$$

Here $k_{1}$ and $k_{3}$ are coefficients associated with the separation distance between the top and the bottom magnets. Xis the base displacement, $\mathrm{y}$ is the displacement of the middle maget, $\mathrm{z}$ is the displacement; $\mathrm{m}_{1}, \mathrm{~m}_{2}$ is the mass of the central magnet and the mass under the spring respectively, $\mathrm{a}$ is the gravitational constant, and $c_{1,} c_{2}$ is the damping coefficients and the damping comes from the frictional contact between the pipe and the moving magnet and air.

In sum, combining (1) yields the electro-magneto-mechanical model of the proposed magneto electric energy harvester. The mechanical response can be obtained by solving these equations.
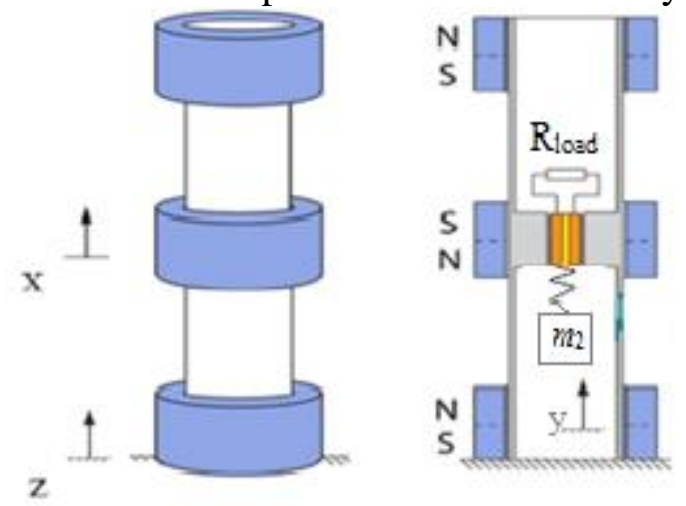

Fig. 1.A schematic diagram of the magnetic levitation energy harvesting system

\section{Harmonic balance analysis}

This section applies the method of harmonic balance to study the magneto electric energy harvester to external harmonic loading. The following analysis is of value in that analytical methods are provided for characterizing the response of the harvester to a wide range of frequencies. Considering the accelerating interest in this type of design, analytical expressions for the magneto electric energy harvester response provide for fast and convenient methods for optimization and preliminary suitability analyses. Furthermore, the response of a strongly nonlinear harvester to a harmonic load is non-trivial and deserving of a more thorough analysis that does not yet exist in the literature. Upon introducing a harmonic load of, the dimensionless equations are modified as

$$
\left\{\begin{array}{l}
x^{\prime \prime}+\gamma_{1} x^{\prime}+x+\beta x^{3}+a_{1}-\mu f \gamma_{2} y^{\prime}-\mu f^{2} y=p \cos \omega t \\
y^{\prime \prime}+(1+\mu) f \gamma_{2} y^{\prime}+(1+\mu) f^{2} y-\gamma_{1} x^{\prime}-x-\beta x^{3}=0
\end{array}\right.
$$

Here the coefficients of Eq. (1) have been rewritten as

$$
\begin{gathered}
\omega_{1}^{2}=\frac{k_{1}}{m_{1}} ; \omega_{2}^{2}=\frac{k_{2}}{m_{2}} ; \mu=\frac{m_{2}}{m_{1}} ; f=\frac{\omega_{2}}{\omega_{1}} ; \\
\omega=\frac{\Omega}{\omega_{1}} ; p=\frac{W}{\omega_{1}^{2}} ; \gamma_{1}=\frac{c_{1}}{m_{1} \omega_{1}} ; \gamma_{2}=\frac{c_{2}}{m_{2} \omega_{2}} ; \\
\beta=\frac{k_{3}}{k_{1}} ; a_{1}=\frac{a}{\omega_{1}^{2}} ; t=\omega_{1} \tau
\end{gathered}
$$


In the method of harmonic balance, the harvester response is presumed to be accurately modeled by a truncated Fourier series, where the number of terms dictates the accuracy of the intended solution. In previous analyses, there was strong interest in the large orbit, well-mixing harmonic solutions. This type of motion maintains a dominant fundamental frequency at the frequency of excitation. Hence, to analytically characterize such motion we assume that the response of the harvester can be modeled as

$$
\begin{aligned}
& x(t)=a_{10}(t)+a_{11}(t) \sin \omega t+b_{11}(t) \cos \omega t, \\
& y(t)=a_{20}(t)+a_{21}(t) \sin \omega t+b_{21}(t) \cos \omega t
\end{aligned}
$$

Substituting the above expressions into the dimensionless system, neglecting higher harmonics and balancing constant terms and those multiplied by $\sin \Omega t$ and $\cos \Omega t$.In steady state, all time derivatives vanish so that we can re-write the mechanical amplitude equations as

$$
\begin{gathered}
a_{10}+\beta a_{10}{ }^{3}+a_{1}-\mu f^{2} a_{20}+\frac{3}{2} \beta a_{10} a_{11}{ }^{2}+\frac{3}{2} \beta a_{10} b_{11}{ }^{2}=0 \\
(1+\mu) f^{2} a_{20}-a_{10}-\beta a_{10}{ }^{3}-\frac{3}{2} \beta a_{10} a_{11}{ }^{2}-\frac{3}{2} \beta a_{10} b_{11}{ }^{2}=0 \\
b_{11}-\omega^{2} b_{11}+\omega \gamma_{1} a_{11}+\beta\left(3 a_{10}{ }^{2} b_{11}+\frac{3}{4} b_{11}{ }^{3}+\frac{3}{4} a_{11}{ }^{2} b_{11}\right)-\mu \omega f \gamma_{2} a_{21}-\mu f^{2} b_{21}-p=0 \\
a_{11}-\omega^{2} a_{11}-\omega \gamma_{1} b_{11}+\beta\left(3 a_{10}{ }^{2} a_{11}+\frac{3}{4} a_{11} b_{11}{ }^{2}+\frac{3}{4} a_{11}{ }^{3}\right)+\mu \omega f \gamma_{2} b_{21}-\mu f^{2} a_{21}=0
\end{gathered}
$$

\section{Simulation and discussion}

In this section, approximate analytical results will be illustrated by numerical examples. In the following numerical case, the magneto electric energy harvester will be considered. If its physical parameters are $m_{1}=0.012 \mathrm{~g}, c_{1}=0.1, k_{1}=6.39 \mathrm{~N} / \mathrm{m}, k_{3}=166000 \mathrm{~N} / \mathrm{m}^{3}, a=3 \mathrm{~m} / \mathrm{s}^{2}, c_{2}=0.01, k_{2}=0.01, m_{2}=0.012 u \mathrm{~g}, \mathrm{u}=0.05$ (it is strongly nonlinear) and substituted into (5-8),we get fig 2 ,relative amplitude response to different frequency; If $u=1$, we get fig 3 , relative amplitude response to different frequency. In fig. 4, the frequency response of middle magnet is showed as the mass ratio $u$ changes The numerical results show that the strength and bandwidth of energy harvesting are improved by adjusting the mass ratio. The analytical results are supported by the numerical solutions.

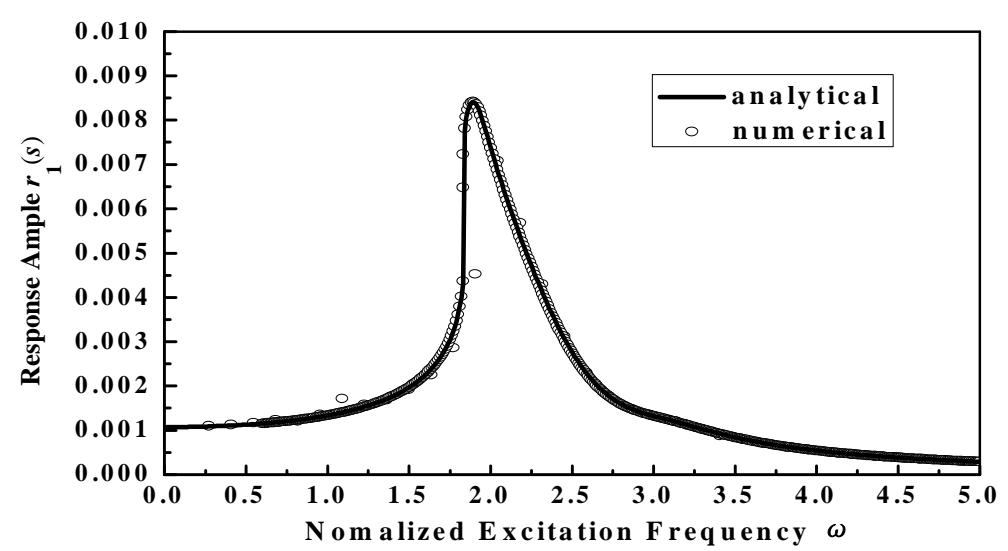

Fig. 2. Frequency response of middle magnet as the mass ratio $\mathrm{u}$ is 0.05 . 


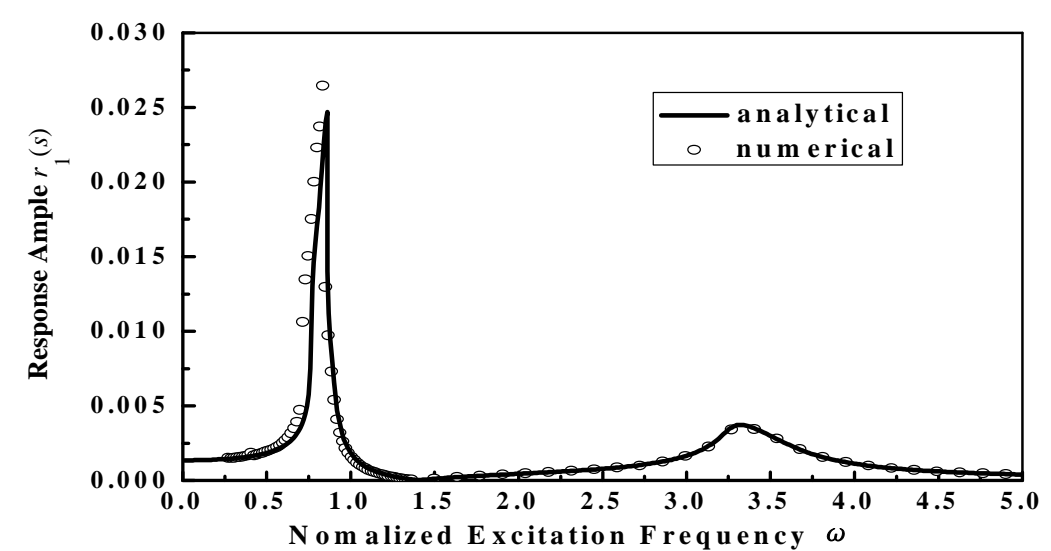

Fig. 3. Frequency response of middle magnet as the mass ratio $\mathrm{u}$ is 1 .

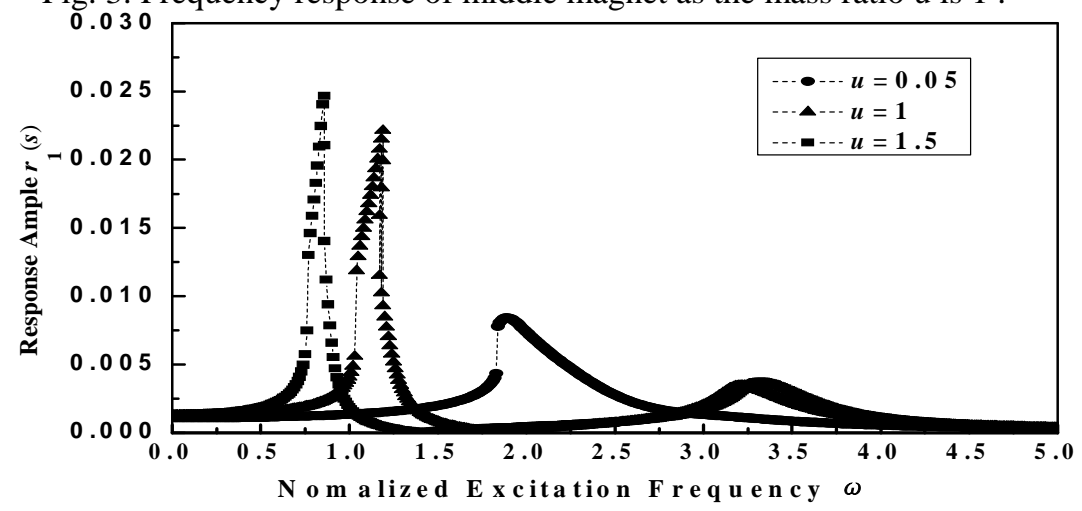

Fig. 4. Frequency response of middle magnet as the mass ratio u changes

\section{References}

[1] N. Elvin, A. Erturk (Eds.),Advances in Energy Harvesting Methods, Springer, New York, 2013.

[2] A. Erturk, D.J. Inman,Piezoelectric Energy Harvesting, John Wiley and Sons Ltd, Wiley, 2011.

[3] H.A. Sodano, G. Park, D.J. Inman, A review of power harvesting from vibration using piezoelectric materials,Shock Vibration Digest36 (2004) 197-205.

[4] S.R. Anton, H.A. Sodano, A review of power harvesting using piezoelectric materials (2003-2006),Smart Material Structures16 (2007) R1-R21.

[5] L.H. Tang, Y.W. Yang, C.K. Soh, Toward broadband vibration-based energy harvesting,Journal of Intelligent Material Systems and Structures 21 (2010) 1867-1897.

[6] C. Nan, M. I. Bichurin, S. Dong, D. Viehland, and G. Srinivasan, Multiferroic magnetoelectric composites: Historical perspective, status, and future directions," J. Appl. Phys. , vol. 103, p. 031101, Feb. 2008.

[7] X. Dai, Y. Wen, P. Li, J. Yang, and G. Zhang, "Modeling, characterization and fabrication of vibration energy harvester using Terfenol-D/ PZ," Sensors Actuators A,vo 1. 156, pp. 350-358, Dec. 2009.

[8] Wu Z., Harne R. L., WangEnergy K. W. Harvester Synthesis Via Coupled Linear-Bistable System With Multistable Dynamics[J]. Journal of Applied Mechanics 2014,81: 0610051-0610059.

[9] P. Li, Y. Wen, P. Liu, X. Li, and C. Jia, “A magnetoelectric energy harvester and management circuit for wireless sensor network, Sensors Actuators A, vol. 157, pp. 100-106, Jan. 2010.

[10] Shengxi Zhou, Junyi Cao, Daniel J. Inman, Jing Lin, Shengsheng Liu, Zezhou Wang. Broadband tristable energy harvester: Modeling and experiment verification [J]. Applied Energy,2014,133:33-39. 
[11] Dai Xianzhi, Wen Yumei, Li Ping, Yang Jin, Zhang Gao -yong. Modeling characterization and fabrication of vibration energy harvester.using Terfenol-D /PZT/ Terfenol-D. composite transducer [J]. Sensors and Actuators, 2009, A156: 350-358.

[12] Wen-An Jiang, Chen Li-Qun. Energy harvesting of monostable Duffing oscillator under Gaussian white noise excitation[J]. Mechanics Research Communications, 2013, 53: 85-91. [13] B.P. Mann , N.D. Sims Energy harvesting from the nonlinear oscillations of magnetic levitation Journal of Sound and Vibration 319 (2009) 515-530

[14] Yang Zhu and Jean W. Zu IEEE TRANSACTIONS ON Magnetics, Vol. 48, NO. 11, November 2012 